\title{
ORIGINAL ARTICLE Heritable variation in maternally derived yolk androgens, thyroid hormones and immune factors
}

\author{
S Ruuskanen ${ }^{1,2}$, P Gienapp ${ }^{2}$, TGG Groothuis ${ }^{3}$, SV Schaper ${ }^{2}$, VM Darras ${ }^{4}$, C Pereira ${ }^{3}$, B de Vries ${ }^{3}$ and \\ ME Visser ${ }^{2,3}$
}

Maternal reproductive investment can critically influence offspring phenotype, and thus these maternal effects are expected to be under strong natural selection. Knowledge on the extent of heritable variation in the physiological mechanisms underlying maternal effects is however limited. In birds, resource allocation to eggs is a key mechanism for mothers to affect their offspring and different components of the egg may or may not be independently adjusted. We studied the heritability of egg components and their genetic and phenotypic covariation in great tits (Parus major), using captive-bred full siblings of wild origin. Egg mass, testosterone ( $\mathrm{T}$ ) and androstenedione (A4) hormone concentrations showed moderate heritability, in agreement with earlier findings. Interestingly, yolk triiodothyronine hormone (T3), but not its precursor, thyroxine hormone (T4), concentration was heritable. An immune factor, albumen lysozyme, showed moderate heritability, but yolk immunoglobulins (IgY) did not. The genetic correlation estimates were moderate but statistically nonsignificant; a trend for a positive genetic correlation was found between A4 and egg mass, T and lysozyme and IgY and lysozyme, respectively. Interestingly, phenotypic correlations were found only between $\mathrm{A} 4$ and $\mathrm{T}$, and $\mathrm{T} 4$ and $\mathrm{T} 3$, respectively. Given that these egg components are associated with fitness-related traits in the offspring (and mother), and that we show that some components are heritable, it opens the possibility that natural selection may shape the rate and direction of phenotypic change via egg composition.

Heredity (2016) 117, 184-190; doi:10.1038/hdy.2016.49; published online 6 July 2016

\section{INTRODUCTION}

Maternal effects are referred as the effect of maternal phenotype on offspring phenotype (Mousseau and Fox, 1998). They make a significant contribution to variation in fitness by modifying offspring phenotype, thereby altering the genotype-phenotype relationship, potentially accelerating or impeding the response to selection on traits affected by maternal investment (for example Kirkpatrick and Lande, 1989; McGlothlin and Galloway, 2014). Maternal genetic effects on offspring traits can arise from among-female genetic variance in, for example, postnatal parental care, or prenatal transfer of resources or signals, such as hormones. However, additive genetic variation in prenatal mechanisms underlying maternal effects has rarely been studied in non-domesticated animals (for example, Wilson and Reale, 2006) and in general, it is rarely studied in physiological traits (especially in wild species, Charmantier et al., 2014).

Oviparous species, such as birds, are especially suitable models for studying genetic variation in prenatal mediators of maternal effects as their eggs develop outside the mother's body, facilitating the measurement of maternal resources and signals at the prenatal stage. Evidently, the size of the propagule or egg is an important mediator of maternal effects; it has a major influence on offspring phenotype and survival (Williams, 2012). Indeed, high heritability of egg size in birds has been reported (reviewed in Williams, 2012). Additionally, other resources in the egg, such as antioxidants, immune factors and signals such as hormones, are now widely acknowledged as crucial for offspring fitness-related traits (McNabb and Wilson, 1997; von Engelhardt and Groothuis, 2011; Grindstaff et al., 2003). Estimating and verifying the genetic variation in the transfer of resources and developmental signals to the egg is important because it is often assumed that it is shaped by natural selection (Müller et al., 2007; Tschirren et al., 2014) and importantly, it could contribute to the evolution of multiple offspring traits. As the physiological mechanisms behind the transfer of different components to eggs as well as their costs (energetic, nutritional and physiological) differ (Groothuis and Schwabl 2008), it is hence crucial to study the extent of genetic variation and covariation in multiple egg components in a single system.

Important developmental signals are egg hormones such as steroid hormones (testosterone, T, and its precursor, androstenedione, A4, von Engelhardt and Groothuis, 2011) and the less-studied thyroid hormones (THs, the bioactive triiodothyronine, T3, and its precursor thyroxine, T4; McNabb and Wilson 1997). Both can affect offspring early development in various taxa (positively or negatively, reviewed by, for example, McNabb and Wilson 1997; Dufty et al., 2002; McNabb 2007; von Engelhardt and Groothuis, 2011), but also phenotype and fitness in adulthood (von Engelhardt and Groothuis, 2011; Ruuskanen et al., 2012; Tschirren et al., 2014). The production

${ }^{1}$ Section of Ecology, Department of Biology, University of Turku, Turku, Finland; ${ }^{2}$ Department of Animal Ecology, Netherlands Institute of Ecology (NIOO-KNAW), Wageningen, The Netherlands; ${ }^{3}$ Groningen Institute for Evolutionary Life Sciences, University of Groningen, Groningen, The Netherlands and ${ }^{4}$ Laboratory of Comparative Endocrinology, Biology Department, KU Leuven, Leuven, Belgium

Correspondence: Dr S Ruuskanen, Section of Ecology, Department of Biology, University of Turku, Turku 20014, Finland.

E-mail: skruus@utu.fi

Received 19 December 2015; revised 29 April 2016; accepted 8 May 2016; published online 6 July 2016 
or deposition of higher levels of hormones to eggs likely has no large energetic or nutritional costs to the mother (Groothuis and Schwabl, 2008, but note the potential costs of iodine-limitation on $\mathrm{TH}$ production), but may show physiological costs to the mother, for example, arising from negative effects of elevated androgen levels in female circulation (see also Tschirren et al., 2014) and contextdependent effects in the offspring (von Engelhardt and Groothuis, 2011). Heritability of yolk androgen concentrations has only been reported in a few studies: lines selected for yolk $\mathrm{T}$ showed moderate realized heritability in captive quail (Coturnix japonica, Okuliarova et al., 2011). In the only study in a wild species, Tschirren et al. (2009), using mother-daughter regressions, reported high heritability for $\mathrm{T}$, but not for A4 in collared flycatchers (Ficedula albicollis). However, similar analyses of yolk $\mathrm{T}$ in captive canaries (Serinus canaria) showed no statistically significant heritability (Müller et al., 2012). In addition, studies on captive great tit (Parus major) lines selected for behavior, $\mathrm{T}$ and A4 levels evolved as a correlated evolutionary response to selection on behavior (Groothuis et al., 2008). These results make yolk androgen hormones good candidates for further exploration of their quantitative genetics. In contrast, yolk THs have been largely neglected in the ecological literature and despite their potential importance for development (Ruuskanen et al., 2016a), data on environmental and genetic variation are lacking.

Mothers also transfer multiple types of energetically or nutritionally costly resources to eggs. These include immune factors, such as immunoglobulins (IgYs) (for example, Grindstaff et al., 2003) and albumen antimicrobial proteins (for example, lysozyme: Saino et al., 2002), which affect hatching success, growth and even survival in birds (reviewed in Grindstaff et al., 2003). Thus parent-offspring conflict and trade-offs between female survival and embryo survival may exist, and selection may be opposite for mothers and offspring. In poultry, artificial selection experiments on immune factors suggest a genetic basis of IgY and lysozyme transfer (Grindstaff et al., 2003; Abdel-Moneim and Abdel-Gawad, 2006; Okuliarova et al., 2014), but there are no studies on their heritability in non-domestic birds.

Importantly, the concentrations of different egg components may not be fully independent, but phenotypically or genetically correlated - the evolutionary potential depends on the genetic covariation with other selected traits (Lande and Arnold, 1983). It has been hypothesized that females balance the costs and benefits of one component with another. For example, higher levels of immune factors could compensate the immunosuppressing effects of high yolk androgen levels (Royle et al., 2001; Groothuis et al., 2006), but the results of the phenotypic correlations are currently contradictory (Groothuis et al.,
2006; Gasparini et al., 2007; Hargitai et al., 2009; Ruuskanen et al., 2011; Postma et al., 2014). On the other hand, yolk hormones A4 and $\mathrm{T}$ that are phenotypically correlated have been found to show differential effects in offspring: $\mathrm{A} 4$, but not $\mathrm{T}$, decreased growth (Muriel et al., 2013) and A4, but not T, was positively correlated with offspring recruitment (Hegyi et al., 2011; Tschirren et al., 2014). Furthermore, high yolk A4 concentrations and low T concentrations were associated with higher maternal lifetime reproductive success (Tschirren et al., 2014); suggesting that selection acts oppositely on the correlated traits, which would constrain adaptive allocation. Currently, to our knowledge, data on genetic correlations between egg hormones and immunofactors are lacking (but see Okuliarova et al., 2014) and such analyses for any physiological traits in the wild (birds) are scarce (Charmantier et al., 2014). All in all, to understand the evolution of multiple (correlated) egg components and the traits affected by this maternal investment, it is important to study the heritability of different egg components, and their genetic and phenotypic covariation, within a single species and environment.

We estimated the heritability in a number of maternally derived egg components, that is, important mediators of avian maternal effects, including yolk androgen hormones (T and A4), THs (T4 and T3), immune factors (total IgY and lysozyme enzyme activity) and egg mass, using a passerine bird model, the great tit (Parus major). Furthermore, we analyzed both genetic and phenotypic correlations among the egg components. We used eggs of captive-bred full siblings of wild origin. In great tits, levels of yolk hormones and egg immune factors vary within and across clutches with environmental and social cues (for example, Groothuis et al., 2008; Remes, 2011; Ruuskanen 2016b), but genetic variation has not yet been studied. Most importantly, many of these egg components have been found to modulate offspring development and phenotype in great tits (Podlas et al., 2013; Ruuskanen et al., 2016a). Especially interesting are the potential effects of yolk androgens on dispersal strategies and personality development in great tits (Tschirren et al., 2007; Groothuis et al., 2008)—understanding the underlying quantitative genetics of yolk androgen levels will thus provide insight into the evolution of these traits.

\section{METHODS}

General protocol and captive rearing

The data collection and experiments are described in detail elsewhere (Schaper et al., 2013). See Figure 1 and Table 1 for the data collection setup and sample sizes. The birds were the offspring wild parental pairs (ca10 pairs per year) from a long-term monitored population at the Hoge Veluwe National Park (the Netherlands), where pedigree information is available. Thus, the females from

\begin{tabular}{|c|c|c|c|c|c|}
\hline \multirow[b]{3}{*}{ Experimental year } & \multicolumn{5}{|c|}{ Chicks born in the wild } \\
\hline & \multicolumn{5}{|c|}{ Chicks brought to captivity on day 10 post-hatching } \\
\hline & 2006 & 2007 & 2008 & 2009 & 2010 \\
\hline \multirow[t]{2}{*}{ \# broods=families brought to captivity } & 9 & 10 & 9 & 14 & 8 \\
\hline & \multicolumn{5}{|c|}{ Females laying eggs in captivity } \\
\hline \multirow[t]{3}{*}{ \# females laying eggs in captivity } & 28 & 24 & 20 & 26 & 15 \\
\hline & & \multicolumn{3}{|c|}{ Eggs collected for analyses } & \\
\hline & 146 & 145 & 113 & 137 & 85 \\
\hline
\end{tabular}

Figure 1 A schematic figure of the experimental design, data collection and sample sizes per experimental year. 
Table 1 Results of the ASReml animal model analyses of egg components of great tits (Parus major)

\begin{tabular}{|c|c|c|c|c|c|c|c|}
\hline & Egg mass (g) & $T\left(p g m g^{-1}\right)$ & $A 4\left(p g m g^{-1}\right)$ & T4 $\left(p g m g^{-1}\right)$ & T3 $\left(p g m g^{-1}\right)$ & Lysozyme activity & $\lg Y\left(U \mathrm{ml}^{-1}\right)$ \\
\hline$V_{\text {ind }}$ & $0.0048(0.0056)$ & $44.1(70.3)$ & $199.6(129.0)$ & $0.0741(0.0235)$ & $4.42 \mathrm{e}-09(5.71 \mathrm{e}-10)$ & $0.0286(0.159)$ & $0.0591(0.0347)$ \\
\hline$V_{a}$ & $0.0152(0.007)$ & $211.0(91.1)$ & $271.6(149.6)$ & 7.22e-08 (9.70e-09) & $0.00443(0.0018)$ & $0.255(0.172)$ & $0.041(0.0379)$ \\
\hline$h^{2}$ & $0.48(0.19)$ & $0.48(0.18)$ & $0.33(0.17)$ & $3.32 \mathrm{e}-07$ (4.37e-08) & $0.25(0.09)$ & $0.20(0.13)$ & $0.25(0.22)$ \\
\hline$x^{2} / t^{a}$ & 7.71 & 9.50 & 6.42 & 0.00 & $2.46^{\mathrm{a}}$ & 3.99 & 1.20 \\
\hline$N$ (females) & 98 & 113 & 111 & 77 & 77 & 111 & 95 \\
\hline$N$ (families) & $26(48)$ & $30(49)$ & $30(49)$ & $21(41)$ & $21(41)$ & $30(49)$ & $25(44)$ \\
\hline Mean (s.e.) & $1.57(0.18)$ & $68(21)$ & $112(29)$ & $1.5(0.5)$ & $0.23(0.13)$ & $10.4(1.4)$ & $141(172)$ \\
\hline range & $1.1-2.4$ & $26-143$ & $38-216$ & $0.4-3.1$ & $0.08-1.12$ & $5-13$ & $4-1384$ \\
\hline
\end{tabular}

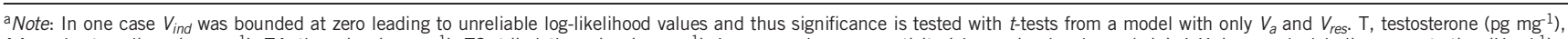
$\mathrm{A} 4$, androstenedione $\left(\mathrm{pg} \mathrm{mg}^{-1}\right), \mathrm{T} 4$, thyroxine $\left(\mathrm{pg} \mathrm{mg}^{-1}\right), \mathrm{T} 3$, triiodothyronine $\left(\mathrm{pg} \mathrm{mg}{ }^{-1}\right)$, Lysozyme, lysozyme activity (change in absorbance/min), IgY, immunologlobulin concentration (U ml-1). Final models including fixed factors are presented Supplementary Table 1 in full. Mean ( \pm s.e.) and range of variation for each egg component is also shown. $N$ (families): the number of families with data from $\geqslant 2$ siblings (and in brackets, the total number of families, as not all siblings laid eggs); $N$ (females) is the number of individual females (=, nb of clutches), $N$ (eggs) is the total number of egg used in the analyses.

Variance components, heritabilities and sample sizes $(N)$ are shown. $V_{\text {ind }}$ phenotypic variance related to individual female, $V_{a}$, additive genetic component, $V_{\text {res, }}$ residual variance. The heritability $\left(h^{2}\right)$ is the proportion of the variance explained by the additive genetic effect $\left(V_{a} V_{p}\right)$. Estimates are followed by their approximate standard errors (in brackets). Chi-square values and significances refer to $V_{a}$ and these tests are likelihood ratio-test of model including effect vs model without effect. Bold values signifies $P<0.05$.

which we analyzed egg composition come from a total of 50 families and some individuals are full-sibs to each other. For the purposes of other studies, parental broods of early and late egg-laying dates were selected. The selected broods had known ancestors, large brood size and a balanced sex ratio. Paternity by the social father was verified using paternity analyses (following Saladin et al., 2003). Chicks were raised in natural nests until day 10 post hatching after which the chicks were taken to captivity (Netherlands Institute of Ecology) as complete broods for hand-rearing under standardized conditions (see Schaper et al., 2013), thus limiting an inflated heritability measure due to common environment effects.

After independence, fledglings were transferred to single-sex groups in open outdoor aviaries. Each year of the 5 study years, 36 breeding pairs were formed avoiding sib-matings. On 1 December, the pairs were placed in climatecontrolled aviaries to breed in the next spring (see Schaper et al., 2013 for details on the aviary conditions). Over 5 experimental years, birds were exposed to varying temperature regimes, which were designed to test temperature effects on the onset of reproduction (Schaper et al., 2013). Sibling females were subject to different temperature treatments. Temperature was included in the statistical analyses (see below). However, temperature treatments/realized temperatures had only a very limited direct effects on the different egg components, except on lysozyme (see Supplementary Table 1). All experimental procedures were approved by the Animal Experimentation Committee (DEC) of the Royal Netherlands Academy of Arts and Sciences (KNAW).

\section{Data collection and sampling}

Nest boxes inside the aviaries were checked daily for eggs, eggs collected on the day of laying, weighed $(\sim 0.001 \mathrm{~g})$ and replaced by artificial eggs. Eggs were stored in $-80^{\circ} \mathrm{C}$ until analyses were performed (see below). Steroid and THs have been found resistant to long-term storage, and our results on hormones and immune parameters are similar as measured in fresh samples (Ruuskanen et al., 2016b) suggesting that any effect of storage time is likely to be small. Additionally, year was controlled in the analyses, but did not have a statistically significant effect (see below).

We included in our data all first clutches with clutch size between 4 and 15 eggs from all experimental years (analysis was restricted to these clutch sizes as they are in the range of normal clutch sizes in Dutch wild populations). Additionally, from clutches with clutch size of 3 eggs ( $N=5$ clutches) or $>15$ eggs ( $N=8$ clutches), we analyzed the third egg as a representative of the clutch mean to increase our sample size (comparison of the clutch mean vs third egg, $r_{s}$ 0.74-0.83, all $P$-values $<0.001, N=74-91$, depending on the egg component). We analyzed all of the eggs in the laying sequence from 10 clutches (divided across years) for yolk A4 and T, lysozyme and egg mass. From the rest of the clutches, we analyzed egg 1 and 3, then every third egg and always the last egg for T, A4, lysozyme and IgY. Only a small sample volume was needed for each of the above components; thus all these components could be measured from a single egg. However, for yolk THs, a complete yolk was needed for the analysis, and thus it could not be analyzed from the same eggs as the other components. We thus analyzed egg 2, 4 and penultimate egg, if available, for THs.

\section{Laboratory analysis methods}

Shell, albumen and yolk were separated by thawing. For the analyses of yolk steroids and IgY the yolk was mixed with water (MilliQ 1:1) with the help of glass beads, and frozen at $-80{ }^{\circ} \mathrm{C}$ until assays were conducted. See Supplementary material for a full description of the analysis methods. Briefly, $T$ and A4 concentrations were analyzed using extractions and radioimmunoassay as described earlier (Ruuskanen et al., 2015), and are expressed as $\mathrm{pg} / \mathrm{mg}$. T4 and T3 concentrations were also analyzed using extractions and radioimmunoassay as described earlier (Ruuskanen et al., 2015) and are expressed as pg/mg. Lysozyme activity was analyzed using a micro-plate modification of a turbidimetric assay, following Ruuskanen et al. (2011), where lysozyme breaks down the bacterial cell walls in the suspension which can be seen by the change in turbidity. The results are expressed as lysozyme activity $=$ change in absorbance $/ \mathrm{min} \times 1000$. Immunoglobulin $($ IgY) concentrations were determined using enzyme-linked immunoassays, following Ruuskanen et al. (2011) and the results are expressed as $\mathrm{Uml}^{-1} \times 10^{5}$.

\section{Statistical analyses}

We first tested which environmental/phenotypic factors explain variation in the levels of egg components, and thus should be accounted for in the quantitative genetic analyses. Our responses were: egg mass, yolk T, A4, T4 and T3 concentration (log-transformed, $\mathrm{pg} \mathrm{mg}^{-1}$ ), yolk IgY concentration (logtransformed, $\mathrm{U} \mathrm{ml} \mathrm{ml}^{-1}$ ) and lysozyme activity (change in absorbance/min). The models included the following fixed effects: proportional egg order and its second-order term $(0=$ first egg, $1=$ last egg, to account for within-clutch variation, taking into account the very variable clutch sizes), size of the female (tarsus), the date when the first egg of a clutch was laid (hereafter: egg-laying date), clutch size, the female's hatching date (that is, early conditions), year and temperature during egg-laying (clutch mean temperature during laying of all eggs, and variation within clutches, that is, deviation of average temperature 4 days before laying each egg from the clutch mean). We included clutch ID as a random intercept to control for non-independence of eggs from the same clutch. In the models for T3, T4, IgY and lysozyme, we also initially included batch number as random effect due to possible variation among assays. 
All models were reduced by removing nonsignificant interactions and main effects, starting from the highest $P$-values. The statistically significant variables retained in the final models were further used in our quantitative genetic analyses. These mixed models (proc MIXED) were conducted with SAS 9.3.

As complete broods were taken into the laboratory for this experiment, quantitative genetic parameters could have been estimated using full-sib analyses. Some parents of these broods were however related and to make use of this additional information we used so-called 'animal models', which utilize such pedigree information (Wilson et al., 2010). The animal model is a mixed model that allows partitioning the total phenotypic variance into several components, which are: the additive genetic variance $\left(V_{A}\right)$, variance due to the non-genetic individual component $\left(V_{\text {ind }}\right)$ and the residual variance $\left(V_{\text {res }}\right)$. Heritability $\left(h^{2}\right)$ and genetic correlations $\left(r_{G}\right)$ were calculated as

$$
h^{2}=\frac{V_{A}}{V_{P}} \text { and } r_{G}=\frac{\operatorname{Cov}_{A}\left(z_{1}, z_{2}\right)}{\sqrt{V_{A}\left(z_{1}\right) V_{A}\left(z_{2}\right)}}
$$

We included all variables (fixed and random) that were retained in the final models above as explanatory variables and added the additive genetic effect as a random effect. The significance of the additive genetic effect was tested using Likelihood ratio-tests with one d.f. The significance of fixed effects was tested with Wald tests with Kenward-Roger adjustment for denDFs. All animal models were run using ASReml-R 3.0.

Finally, we analyzed genetic covariances and correlations between the egg components that showed at least moderate heritabilities (even if not statistically significant) as traits that show no genetic variation cannot—by logic — show any genetic covariation, either. Genetic covariances were estimated with bivariate animal models. To simplify the models and thereby improve model fit, we averaged egg components over clutches (correcting for significant fixed effects). Since this yielded a single clutch mean per individual we could drop the individual random effect from the bivariate models, which hence separated (co) variances into additive genetic and residual component only. The significance of the genetic covariance was tested with Likelihood ratio-tests with one d.f. by comparing full models against models in which the genetic covariance was constrained to be zero. Likelihood-ratio test statistic is calculated as two times the difference in log-likelihoods of models and tested against Chi-square distribution. To study phenotypic correlations, we calculated Pearson $r$ using clutch mean values.

\section{RESULTS}

Mean values and variation in the raw data for each egg component are presented in Table 1. The variance components from 'animal model' analyses are presented in Table 1 and final, reduced models including the fixed factors are in Supplementary Table 1 in full. We found that egg mass was highly heritable $\left(h^{2}=0.48\right.$, Table 1$)$. Both yolk T and A4 concentrations were highly/moderately heritable (T: $h^{2}=0.48$, A4: $h^{2}=0.33$, Table 1). Furthermore, yolk T3 concentration showed low $\left(h^{2}=0.25\right)$, but statistically significant heritability, while T4 concentration was not heritable $\left(h^{2}<0.01\right.$, Table 1$)$. Considering the egg immune factors, albumen lysozyme activity had low $\left(h^{2}=0.20\right)$ and significant heritable variation, while the heritability of $\operatorname{IgY}$ was similar $\left(h^{2}=0.25\right)$ but statistically not significant (Table 1). Most of the egg components varied across the laying order (statistically significant effects of proportional egg number and/or its quadratic term), but there were very few strong associations between individual or environmental variation and egg composition (Supplementary Table 1).

We found a moderate, but statistically nonsignificant, positive genetic correlation between $\mathrm{T}$ and $\mathrm{A} 4$ concentration, and $\mathrm{A} 4$ concentration and egg mass (Table 2). We also found positive phenotypic correlations between $\mathrm{T}$ and $\mathrm{A} 4$, and A4 and egg mass (Table 3 ). T concentration and lysozyme activity showed a marginally

Table 2 Genetic covariances and correlations among the egg components in great tits (Parus major), using clutch mean values

\begin{tabular}{|c|c|c|c|c|c|c|}
\hline $\mathrm{T}$ & - & $\begin{array}{c}0.56 \pm 0.24 \\
N=111\end{array}$ & $\begin{array}{c}0.46 \pm 0.39 \\
N=77\end{array}$ & $\begin{array}{c}-0.12 \pm 0.41 \\
N=95\end{array}$ & $\begin{array}{c}0.61 \pm 0.36 \\
N=111\end{array}$ & $\begin{array}{c}0.33 \pm 0.34 \\
N=98\end{array}$ \\
\hline T3 & $\begin{array}{c}0.508 \pm 0.436 \\
X^{2}=1.52, P=0.22\end{array}$ & $\begin{array}{c}0.379 \pm 0.543 \\
X^{2}=0.56, P=0.45\end{array}$ & - & $\begin{array}{c}0.03 \pm 0.52 \\
N=67\end{array}$ & $\begin{array}{c}0.42 \pm 0.49 \\
N=77\end{array}$ & $\begin{array}{c}0.34 \pm 0.43 \\
N=77\end{array}$ \\
\hline Lysozyme & $\begin{array}{c}6.89 \pm 4.09 \\
X^{2}=3.00, P=0.08\end{array}$ & $\begin{array}{c}1.16 \pm 5.29 \\
X^{2}=0.05, P=0.82\end{array}$ & $\begin{array}{c}0.025 \pm 0.029 \\
X^{2}=0.82, P=0.37\end{array}$ & $\begin{array}{c}0.146 \pm 0.081 \\
X^{2}=3.60, P=0.06\end{array}$ & - & $\begin{array}{c}-0.53 \pm 0.41 \\
N=98\end{array}$ \\
\hline Egg mass & $\begin{array}{c}0.535 \pm 0.551 \\
X^{2}=1.05, P=0.31\end{array}$ & $\begin{array}{c}1.18 \pm 0.70 \\
X^{2}=3.70, P=0.06\end{array}$ & $\begin{array}{l}0.0038 \pm 0.0043 \\
X^{2}=\mathrm{NA}, P=\mathrm{NA}^{\mathrm{a}}\end{array}$ & $\begin{array}{c}0.0061 \pm 0.0109 \\
X^{2}=0.35, P=0.56\end{array}$ & $\begin{array}{c}-0.046 \pm 0.036 \\
X^{2}=1.86, P=0.17\end{array}$ & - \\
\hline
\end{tabular}

Covariances are given in the lower off-diagonals and genetic correlations in the upper off-diagonals. Estimates \pm s.e.s, and test statistics for covariances are presented. Sample sizes $(M)$ are indicated below. T, testosterone $\left(\mathrm{pg} \mathrm{mg}^{-1}\right), \mathrm{A} 4$, androstenedione $\left(\mathrm{pg} \mathrm{mg}^{-1}\right), \mathrm{T}$, triiodothyronine $\left(\mathrm{pg} \mathrm{mg}^{-1}\right)$, Lysozyme, lysozyme activity (change in absorbance/min), IgY, immunoglobulin concentration $\left(\mathrm{U} \mathrm{ml}^{-1}\right)$. a Model likelihood did not converge; therefore, LRT against constrained model not informative.

Table 3 Phenotypic correlations (Pearson's $r$ ) among the egg components in great tits (Parus major), using clutch mean values

\begin{tabular}{|c|c|c|c|c|c|c|}
\hline & Egg mass & $T$ & A4 & T3 & T4 & $\lg Y$ \\
\hline $\mathrm{T}$ & $0.12(N=98)$ & & & & & \\
\hline A4 & $0.21 *(N=97)$ & $0.59^{* * *}(N=111)$ & & & & \\
\hline T3 & $0.13(N=77)$ & $0.14(N=77)$ & $-0.11(N=76)$ & & & \\
\hline $\mathrm{T} 4$ & $-0.04(N=77)$ & $-0.19(N=77)$ & $-0.17(N=76)$ & $0.27^{*}(N=77)$ & & \\
\hline $\lg Y$ & $-0.15(N=82)$ & $0.07(N=95)$ & $0.05(N=94)$ & $0.11(N=67)$ & $0.16(N=67)$ & \\
\hline Lysozyme & $-0.11(N=98$ & $-0.01(N=111)$ & $-0.08(N=110)$ & $0.03(N=77)$ & $-0.03(N=77)$ & $0.16(N=94)$ \\
\hline
\end{tabular}

$* * * P<0.001,{ }^{* *} P<0.01,{ }^{*} P<0.05$.

$\mathrm{T}$, testosterone $\left(\mathrm{pg} \mathrm{mg}^{-1}\right), \mathrm{A} 4$, androstenedione $\left(\mathrm{pg} \mathrm{mg}^{-1}\right), \mathrm{T} 4$, thyroxine $\left(\mathrm{pg} \mathrm{mg}^{-1}\right), \mathrm{T} 3$, triiodothyronine $\left(\mathrm{pg} \mathrm{mg}^{-1}\right)$, Lysozyme, lysozyme activity (change in absorbance/min), IgY, immunologlobulin concentration $\left(\mathrm{U} \mathrm{ml}^{-1}\right)$. Sample sizes (number of clutches) are indicated in parentheses. 
statistically significant, positive genetic correlation (Table 2), but no phenotypic correlation (Table 3). There were no other genetic or phenotypic correlations among any hormones and immune factors (Tables 2 and 3). T3 and T4 were positively phenotypically correlated (Table 3), but T3 showed no genotypic correlation with any of the egg traits (Table 2). Finally, IgY and lysozyme activity showed a moderate, but statistically nonsignificant, positive genetic correlation (Table 2), but no phenotypic correlation (Table 3).

\section{DISCUSSION}

As expected, egg mass was heritable $\left(h^{2}=0.48\right)$, but somewhat lower than published for this species previously $\left(h^{2}=0.66-0.86\right.$, reviewed in Williams 2012). We found moderate heritability for yolk T concentration, which is consistent with previous studies showing significant heritability for $\mathrm{T}$ from lines selected for yolk $\mathrm{T}$ in captive quails $\left(h^{2}=0.42\right.$; Okuliarova et al., 2011) and mother-daughter correlations from wild collared flycatchers $\left(h^{2}=0.75\right.$; Tschirren et al., 2009). Furthermore, in contrast to studies in the wild collared flycatcher, where yolk A4 concentration was not heritable (Tschirren et al., 2009), but consistent with the study in quails (Okuliarova et al., 2011), we found moderate heritability also for yolk A4 concentration. Similarly to our study, also in captive great tit lines selected for personality, co-selection for both yolk $\mathrm{T}$ and A4 concentrations was confirmed (Groothuis et al., 2008). Given that in great tits yolk $\mathrm{T}$ levels affect offspring growth and sibling competition (Podlas et al., 2013) and dispersal distances (Tschirren et al., 2007), all of which are likely to be related to offspring fitness, our results suggest there may be potential for selection, and a response to selection, on yolk androgen levels in great tits. Importantly, recent correlative and experimental studies have reported associations between yolk androgen levels and offspring local survival in other passerine species (Hegyi et al., 2011; Ruuskanen et al., 2012; Tschirren et al., 2014). However, while most studies so far focused on the effects of yolk androgens on the offspring, the fitness effects, costs and benefits associated with differential yolk androgen deposition for the mother have received less attention (for example, Müller et al., 2007), although this is crucial for understanding their evolution. Tschirren et al. (2014) recently reported a positive correlation between lifetime reproductive success and yolk A4, but a negative with T. Furthermore, female lifespan was positively correlated with A4 and negatively with T (Tschirren et al., 2014), suggesting yet unknown costs to the female. Unfortunately, such data from our study species are lacking. Nevertheless, the accumulating evidence for genetic variation and fitness effects of androgen deposition (on offspring and mothers) thus suggest that this can be under selection.

It is also interesting to speculate about the effect of heritable yolk androgen levels for trait evolution: In great tits, the evolution of personality, that is, consistent behavioral differences, known to affect fitness (Groothuis et al., 2008), could be affected by maternal effects. Yolk androgens affect behavior and personality traits (neophobia, aggression, habituation, for example, Daisley et al., 2005; Tobler and Sandell, 2007; Ruuskanen et al., 2012). In great tits, personality further shows a small maternal genetic component (van Oers et al., 2004). Thus, heritable variation in yolk androgen levels may contribute to the heritability and variation in personality, and the rate of evolution of personality. Another potential trait under maternal influence is dispersal: in great tits higher yolk hormone levels are linked to longer natal dispersal distances (Tschirren et al., 2007). If heritable variation in yolk androgen transfer plays a role in evolution of dispersal distances/strategies, it could further affect the population structure or colonization processes.
We found moderate heritability for yolk T3 concentration. There is no prior data on heritability of yolk THs; the only available relevant data show that there is consistent among-female variation in quail yolk T4 levels (McNabb and Wilson, 1997). Yolk THs enter the ovum from the female circulation and are assumed to reflect circulating THs (although possibly with some control, suggested by their nonlinear correlation; McNabb and Wilson, 1997; Groothuis and Schwabl, 2008). There is evidence for heritability of thyroid function and circulating $\mathrm{TH}$ levels in adult vertebrates, including chickens (for example, McLachlan et al., 2011; Loyau et al., 2013). Thus it remains an open question whether heritability of yolk THs simply reflect consistent variation in female circulating T3 levels, or whether yolk T3 levels can evolve independently. Selection experiments would be needed to confirm this (see, for example, Okuliarova et al., 2011 for independent effects of selection on circulating and yolk T). Interestingly, T4 was not heritable. These results suggest that environmental influences are mainly responsible for the variation in yolk T4 levels. These findings are supported by among- and within-clutch variation in relation to temperature and laying date in the study species in yolk T4 but not in T3 (Ruuskanen et al., 2015, 2016b). As T4 is the prohormone for its biologically active metabolite T3, the data suggest that genetic variation in enzymes for the conversion of T4 to T3 and/ or enzymes for T3 degradation exists (circulating T3 levels are dependent on both T3 production and degradation, McNabb, 2007). We can also hypothesize that as T3 is the biologically active form, its regulation to the egg is more important, and multiple mechanisms may have evolved to keep levels constant (independent of variation in circulating T3 levels). However we found a phenotypic correlation between T4 and T3, suggesting constraints on the independent or metabolism transfer of the two hormones. In any case, a major question is whether variation in yolk $\mathrm{T} 3$ has fitness consequences on offspring or the mother, which selection could act on. Unfortunately, the data are currently very limited; in the studied species, experimentally elevated yolk THs (T4 and T3 combined) affected growth, but not fledging success (Ruuskanen et al., 2016a). More studies are needed to elucidate the environmental and genetic contributions and functional significance of yolk THs and any role in trait evolution.

Concerning the egg immune factors, we found significant heritability for egg lysozyme activity. Yolk IgY concentration showed a tendency for low to moderate heritability but this was not statistically significant. Previous data from poultry and quail suggest a heritable basis for maternal IgY and lysozyme transfer (Grindstaff et al., 2003; Abdel-Moneim and Abdel-Gawad, 2006; Okuliarova et al., 2014). The lack of significant heritability for yolk IgY concentration may be due to lack of power (given the large s.e.s). In contrast to hormones, the production of which and transfer to egg is likely not energetically/ nutritionally costly, production and deposition of IgYs into eggs is potentially condition/resource dependent and represents a cost- thus a parent-offspring conflict arises. For example, Kowalczyk et al. (1985) estimated that egg IgYs would reflect $10-20 \%$ of the female IgY production. However for lysozyme the costs are not clear and it likely does not derive from maternal circulation, as IgYs (see discussion in, for example, Shawkey et al., 2008). Lysozyme levels correlate with, for example, hatching success (Saino et al., 2002), but experimental evidence for their fitness effects is lacking, and more research is thus needed to understand their potential for being under selection.

Furthermore, we were interested in the genetic and phenotypic covariation among different egg traits, to investigate whether their transfer may be independent or correlated. The estimates of our genetic correlations are moderate, but nonsignificant, likely due to limited sample size. Reliably estimating covariances typically requires 
more data than estimating variances. However, admitting these limitations in our data, we can still speculate about some interesting patterns that emerged: We found moderate but statistically nonsignificant positive genetic correlation and a positive phenotypic correlation between $\mathrm{T}$ and $\mathrm{A} 4$ concentrations, which suggests lack of independent regulation of the levels of the two hormones in eggs. The correlated selection in A4 to selection for $\mathrm{T}$ in captive quails (Okuliarova et al., 2011) corroborates our findings. Given the recent findings on differential effects of $\mathrm{A} 4$ and $\mathrm{T}$ on offspring development and maternal fitness (for example, Hegyi et al., 2011; Muriel et al., 2013; Tschirren et al., 2014, see introduction), these results suggest that there may be genetic constraints limiting the response to selection, potentially caused by inflexibility in the physiological mechanism (both originate from the ovaries, A4 being a precursor for T, Groothuis and Schwabl, 2008). Unfortunately, the differential effects of each hormone are not known for the study species, and thus the potential evolutionary constraints in great tits are not well understood.

In contrast to our predictions, we found weak or no genetic or phenotypic correlation between androgens and immunofactors. Thus these results provide only weak support for the adaptive covariation hypothesis (Royle et al., 2001; Groothuis et al., 2006). Previous results on phenotypic variation are controversial; in the study species, a phenotypic correlation between androgens and immune factors was found at between- but not within-clutch level (Postma et al., 2014, see also Gasparini et al., 2007), but multiple other studies find no support for the hypothesis (Groothuis et al., 2006; Hargitai et al., 2009; Ruuskanen et al., 2011). In selection lines for yolk T and behavior (quails, Okuliarova et al., 2014), IgY or lysozyme levels did not respond to selection similarly as $\mathrm{T}$, suggesting no genetic covariation. Thus, a similar (or opposite) response to environmental and social conditions could explain the reported phenotypic correlation between yolk androgens and immune factors also in the study species. However, a lack of genetic correlation also allows flexibility to modulate, for example, offspring growth trajectories (within and among clutches), for example in response to environmental context.

Importantly, concerning all the results, we need to consider that birds were hand-reared from day 10 after hatching; thus we cannot fully exclude the effects of similar rearing environment within a nest in early development from genetic effects (Merilä and Sheldon, 2000) or a priming maternal effect, that is, an effect of hormone levels in the egg on the function and development of the female hypothalamusgonadal axis, which then may further affect hormone allocation (Groothuis and Schwabl, 2008). If present, it is possible that these somewhat bias our heritability estimates upwards. However, yolk androgen levels of experimentally in ovo manipulated canary females showed no evidence for such priming effects (Müller et al., 2011). Furthermore, the fact that the birds were reared in captivity reduces the effect of a common (brood) environment on siblings that can inflate heritability estimates. However, the effect of captive conditions may alter (increase or decrease) the phenotypic variation, which may then affect the size of the heritability estimate. Finally, we should mention that our sample size is not very large in respect to traditional quantitative genetic analyses; however, similar sample sizes did show significant heritabilities in a previous study on yolk testosterone (52 mother-daughter pairs, Tschirren et al., 2009).

Finally, in addition to the clutch mean levels, all of the abovementioned egg components have been found to show distinct withinclutch patterns, that is, increase or decrease with laying order, also in the studied species (Groothuis et al., 2008; Ruuskanen et al., 2016b), as found also in this study. There are multiple mutually non-exclusive explanations for such patterns, such as trade-offs between resource allocation between self and eggs, but also potentially adaptive mechanisms, affecting sibling competition (for example, von Engelhardt and Groothuis, 2011). There is some evidence of repeatability of the within-clutch patterns, but also environmental conditions can affect these patterns (von Engelhardt and Groothuis, 2011); thus it would also be interesting to estimate heritability of the within-clutch patterns.

In conclusion, we found moderate heritability of multiple mediators of maternal effects: egg mass, yolk T, A4, T3 and lysozyme, but not IgY or T4, of which many have not been reported previously. Especially interesting are T3 and its precursor T4 which showed a phenotypic correlation, but different degrees of heritability. Our results suggest that both maternal signals (hormones) and resources (immune factors) show genetic variation. Our results indicated a trend for a positive genetic and phenotypic correlation between A4 and T, suggesting constraints for their independent deposition and evolution. Yolk androgens and immune factors showed weak genetic or phenotypic correlations, suggesting that these egg components may evolve independently, and our results do not support the hypothesis of mutual adjustment. Establishing a genetic basis in the pathways mediating maternal effects is required to interpret these as potentially adaptive, and also opens up the opportunity for (hormone-mediated) maternal effects to respond to selection and thus to evolve. Such indirect genetic effects can modify or accelerate phenotypic change in natural populations. In great tits this may particularly be related to personality and dispersal strategies.

\section{DATA ARCHIVING}

Data available from the Dryad Digital Repository: http://dx.doi.org/ 10.5061/dryad.vr666.

\section{CONFLICT OF INTEREST}

The authors declare no conflict of interest.

\section{ACKNOWLEDGEMENTS}

We thank all animal caretakers and field assistants, and laboratory personnel especially Lut Noterdaeme. This study was financially supported by Academy of Finland (to SR, project grant 258419). MEV was supported by an NWOVICI grant.

Abdel-Moneim AS, Abdel-Gawad MMA (2006). Genetic variations in maternal transfer and immune responsiveness to infectious bursal disease virus. Vet Microbiol 114: 16-24. Charmantier A, Garant D, Kruuk LEB (2014). Quantitative Genetics in the Wild. Oxford University press: Oxford, UK.

Daisley JN, Bromundt V, Mostl E, Kotrschal K (2005). Enhanced yolk testosterone influences behavioral phenotype independent of sex in Japanese quail chicks Coturnix japonica. Horm Behav 47: 185-194.

Dufty AM, Clobert J, Møller AP (2002). Hormones, developmental plasticity and adaptation. Trends Ecol Evol 17: 190-196.

Gasparini J, Boulinier T, Gill TA, Gil D, Hatch SA, Roulin A (2007). Food availability affects the maternal transfer of androgens and antibodies into eggs of a colonial seabird. J Evol Biol 20: 874-880.

Grindstaff JL, Brodie ED, Ketterson ED (2003). Immune function across generations: integrating mechanism and evolutionary process in maternal antibody transmission. Proc $R$ Soc Lond B Biol Sci 270: 2309-2319.

Groothuis TGG, Carere C, Lipar J, Drent PJ, Schwabl H (2008). Selection on personality in a songbird affects maternal hormone levels tuned to its effect on timing of reproduction. Biol Lett 4: 465-467.

Groothuis TGG, Eising CM, Blount JD, Surai P, Apanius V, Dijkstra C et al. (2006). Multiple pathways of maternal effects in black-headed gull eggs: constraint and adaptive compensatory adjustment. J Evol Biol 19: 1304-1313.

Groothuis TGG, Schwabl H (2008). Hormone-mediated maternal effects in birds: mechanisms matter but what do we know of them? Phil Trans $R$ Soc B Biol Sci 363: 1647-1661. 
Hargitai R, Arnold KE, Herenyi M, Prechl J, Török J (2009). Egg composition in relation to social environment and maternal physiological condition in the collared flycatcher. Behav Ecol Sociobiol 63: 869-882.

Hegyi G, Herenyi M, Szöllosi E, Rosivall B, Török J, Groothuis TGG (2011). Yolk androstenedione, but not testosterone, predicts offspring fate and reflects parental quality. Behav Ecol 22: 29-38.

Kirkpatrick M, Lande R (1989). The evolution of maternal characters. Evolution 43 : 485-503.

Kowalczyk K, Daiss J, Halpern J, Roth TF (1985). Quantitation of maternal-fetal Igg transport in the chicken. Immunology 54: 755-762.

Lande R, Arnold SJ (1983). The measurement of selection on correlated characters. Evolution 37: 1210-1226.

Loyau T, Berri C, Bedrani L, Metayer-Coustard S, Praud C, Duclos MJ et al. (2013). Thermal manipulation of the embryo modifies the physiology and body composition of broiler chickens reared in floor pens without affecting breast meat processing quality. J Anim Sci 91: 3674-3685.

McGlothlin JW, Galloway LF (2014). The contribution of maternal effects ot selection response: an empirical test of competing models. Evolution 68: 549-558.

McLachlan SM, Lu L, Aliesky HA, Williams RW, Rapoport B (2011). Distinct genetic signatures for variability in total and free serum thyroxine levels in four sets of recombinant inbred mice. Endocrinology 152: 1172-1179.

McNabb FMA (2007). The hypothalamic-pituitary-thyroid (HPT) axis in birds and its role in bird development and reproduction. Crit Rev Toxicol 37: 163-193.

McNabb FMA, Wilson CM (1997). Thyroid hormone deposition in avian eggs and effects on embryonic development. Am Zool 37: 553-560.

Merilä J, Sheldon BC (2000). Lifetime reproductive success and heritability in nature. Am Nat 155: 301-310.

Mousseau TA, Fox CW (1998). Maternal Effects as Adaptations. Oxford University press.

Muriel J, Perez-Rodriguez L, Puerta M, Gil D (2013). Differential effects of yolk testosterone and androstenedione in embryo development and nestling growth in the spotless starling (Sturnus unicolor). Gen Comp Endocrinol 194: 175-182.

Müller W, Goerlich VC, Vergauwen J, Groothuis TGG, Eens M (2012). Sources of variation in yolk hormone deposition: consistency, inheritance and developmental effects. Gen Comp Endocrinol 175: 337-343.

Müller W, Groothuis TGG, Goerlich VC, Eens M (2011). GnRH—a missing link between testosterone concentrations in yolk and plasma and its intergenerational effects. Plos One 6: e22675.

Müller W, Lessells CM, Korsten P, von Engelhardt N (2007). Manipulative signals in family conflict? On the function of maternal yolk hormones in birds. Am Nat 169: E84-E96.

Okuliarova M, Groothuis TGG, Skrobanek P, Zeman M (2011). Experimental evidence for genetic heritability of maternal hormone transfer to offspring. Am Nat 177: 824-834.

Okuliarova M, Kankova Z, Bertin A, Leterrier C, Möstl E, Zeman M (2014). Maternally derived egg hormones, antibodies and antimicrobial proteins: common and different pathways of maternal effects in Japanese quail. PloS One 9: e112817.

Podlas K, Helfenstein F, Richner H (2013). Brood reduction via intra-clutch variation in testosterone - an experimental test in the great tit. PLOS One 8: e56672.

Postma E, Siitari H, Schwabl H, Richner H, Tschirren B (2014). The multivariate egg: quantifying within- and among-clutch correlations between maternally derived yolk immunoglobulins and yolk androgens using multivariate mixed models. Oecologia 174 $631-638$.
Remes V (2011). Yolk androgens in great tit eggs are related to male attractiveness, breeding density and territory quality. Behav Ecol Sociobiol 65: 1257-1266.

Royle NJ, Surai PF, Hartley IR (2001). Maternally derived androgens and antioxidants in bird eggs: complementary but opposing effects? Behav Ecol 12: 381-385.

Ruuskanen S, Darras VM, de Vries B, Visser ME, Groothuis T (2015). Experimental manipulation of food availability leads to short-term intra-clutch adjustment in egg mass but not in yolk androgen or thyroid hormones. J Avian Biol 47: 36-46.

Ruuskanen S, Darras VM, Visser ME, Groothuis TGG (2016a). Effects of experimentally manipulated yolk thyroid hormone levels on offspring development in a wild bird species. Horm Behav 81: 38-44.

Ruuskanen S, Groothuis TGG, Schaper SV, Darras VM, Vries B, Visser ME (2016b). Temperature-induced variation in yolk androgen and thyroid hormone levels in avian eggs. Gen Comp Endocrinol 235: 29-37.

Ruuskanen S, Doligez B, Pitala N, Gustafsson L, Laaksonen T (2012). Long-term fitness consequences of high yolk androgen levels: sons pay the costs. Funct Ecol 26: 884-894.

Ruuskanen S, Siitari H, Eeva T, Belskii E, Järvinen A, Kerimov A et al. (2011). Geographical variation in egg mass and egg content in a passerine bird. Plos One 6.

Saino N, Dall'ara P, Martinelli R, Møller AP (2002). Early maternal effects and antibacterial immune factors in the eggs, nestlings and adults of the barn swallow. J Evol Biol 15: 735-743.

Saladin V, Bonfils D, Binz T, Richner H (2003). Isolation and characterization of 16 microsatellite loci in the Great Tit Parus major. Mol Ecol Notes 3: 520-522.

Schaper SV, Gienapp P, Dawson A, Visser ME (2013). Heritability of gonad size varies across season in a wild songbird. J Evol Biol 26: 2739-2745.

Shawkey MD, Kosciuch KL, Liu M, Rohwer FC, Loos ER, Wang JM et al. (2008). Do birds differentially distribute antimicrobial proteins within clutches of eggs? Behav Ecol 19: 920-927.

Tobler M, Sandell MI (2007). Yolk testosterone modulates persistence of neophobic responses in adult zebra finches, Taeniopygia guttata. Horm Behav 52: $640-645$.

Tschirren B, Fitze PS, Richner H (2007). Maternal modulation of natal dispersal in a passerine bird: an adaptive strategy to cope with parasitism? Am Nat 169: 87-93.

Tschirren B, Postma E, Gustafsson L, Groothuis TGG, Doligez B (2014). Natural selection acts in opposite ways on correlated hormonal mediators of prenatal maternal effects in a wild bird population. Ecol Lett 17: 1310-1315.

Tschirren B, Sendecka J, Groothuis TGG, Gustafsson L, Doligez B (2009). Heritable variation in maternal yolk hormone transfer in a wild bird population. Am Nat 174: 557-564.

van Oers K, Drent PJ, de Jong G, van Noordwijk AJ (2004). Additive and nonadditive genetic variation in avian personality traits. Heredity 93: 496-503.

von Engelhardt N, Groothuis TGG (2011). Maternal hormones in avian eggs. In: Lopez KH, Norris DO (eds) Hormones and Reproduction of Vertebrates: Birds. Academic Press: New York. pp 91-127.

Williams T (2012). Physiological Adaptations for Breeding in Birds. Princeton University Press: USA

Wilson AJ, Reale D (2006). Ontogeny of additive and maternal genetic effects: lessons from domestic mammals. Am Nat 167: E23-E38.

Wilson AJ, Reale D, Clements MN, Morrissey MM, Postma E, Walling CA et al. (2010). An ecologist's guide to the animal model. J Anim Ecol 79: 13-26.

Supplementary Information accompanies this paper on Heredity website (http://www.nature.com/hdy) 